\title{
Geometrical haptic illusions: The role of exploration in the Müller-Lyer, vertical-horizontal, and Delboeuf illusions
}

\author{
EDOUARD GENTAZ \\ Université René Descartes, Paris V, and CNRS, Paris, France \\ and \\ YVETTE HATWELL \\ Université Pierre Mendès-France, Grenoble, France
}

\begin{abstract}
This article surveys studies of the occurrence, in the haptic modality, of three geometrical illusions well known in vision, and it discusses the nature of the processes underlying these haptic illusions. We argue that the apparently contradictory results found in the literature concerning them may be explained, at least partially, by the characteristics of manual exploratory movements. The Müller-Lyer illusion is present in vision and in haptics and seems to be the result of similar processes in the two modalities. The vertical-horizontal illusion also exists in vision and haptics but is due partly to similar processes (bisection) and partly to processes specific to each modality (anisotropy of the visual field and overestimation of radial vs. tangential manual exploratory movements). The Delboeuf illusion seems to occur only in vision, probably because exploration by the index finger may exclude the misleading context from tactile perception. The role of these haptic exploratory movements may explain why haptics is as sensitive as vision to certain illusions and less sensitive to others.
\end{abstract}

For centuries, perceptual illusions (then called geometrical optical illusions) were thought to concern only the visual system and to be due to some specific properties of this system (for a review, see Eagleman, 2001). The question as to whether these illusions also occur in touch was asked during the first half of the 20th century, in particular by the Gestalt psychologists. These researchers maintained that systematic errors relating to the estimation of the size or shape of certain figures were not linked to one particular sense but were a result of the functioning of the nervous system itself and particularly of the interactions (called field effects) between the different parts of the figure. Since, according to Gestalt psychologists, the same processing rules are at work in all the perceptual modalities, illusions analogous to visual ones must also be present in touch. Indeed, this was observed by Revesz (1934) and Bean (1938), who found that almost all the visual illusions existed in a tactual mode. However, since the 1960s, further studies with better methodological control produced sometimes contradictory results, which will be discussed here.

Contemporary studies of the haptic illusions that correspond to visual illusions are doubly interesting from a the-

Correspondence concerning this article should be addressed to E. Gentaz, Laboratory of Cognition and Development (UMR 8605, CNRS), Université René Descartes, 71 avenue Edouard Vaillant, 92774 Boulogne-Billancourt Cedex, Paris, France (e-mail: gentaz@ psycho.univ-paris5.fr). oretical point of view (Gentaz \& Hatwell, 2002). First, they allow the testing of some theories concerning visual illusions and confront purely visual explanations of the phenomenon with non-modality-specific theories. They can also answer the question as to whether the perceptual processes implemented in tactual perception are similar or not to those implemented in visual perception (for reviews, see Hatwell, Streri, \& Gentaz, 2000, 2003; Heller, 2000; Millar, 1994). Thus, not finding a visual illusion in touch is an argument in favor of specific haptic perceptual processes. However, observing the same illusion in vision and touch does not indicate whether the error is a result of similar and/or specific perceptual processes. To do that, we need to know whether the factors responsible for the presence and the variations in intensity of the tactual illusion are identical to those affecting the same phenomenon in vision. An affirmative answer would entail arguments in favor of similar visual and haptic processes, whereas a negative answer would favor specific visual and haptic functioning. In the latter case, the problem would be to identify these modality-specific processes.

In order to answer these questions, three populations are generally compared: blindfolded sighted subjects, subjects who became blind late, and congenitally blind subjects. However, comparison of these may be difficult because of the small number of congenitally blind people and the problems involved in constituting equivalent groups. These difficulties may account for some of the contradictory results found in the literature. 
Blindfolded sighted subjects perceiving manually the spatial properties of objects may use spatial visual representations (see Cornoldi, Bertuccelli, Rocchi, \& Sbrana, 1993; Revesz, 1950; Worchel, 1951). Since visual perception and visual mental images are generally more efficient than haptic perception and nonvisual images in representing space, blindfolded sighted subjects have an advantage over subjects who became blind early (for reviews, see Hatwell, 1978, 1966/1985, 2003; Pick, 1974; Thinus-Blanc \& Gaunet, 1997). But congenitally blind people benefit from greater tactile practice, and this gives them an advantage over the blindfolded sighted. Finally, subjects who became blind late benefit from both spatial visual representations and tactual practice.

If an illusion is present only in the late blind but is absent in the early blind, it can be assumed that visual experience is responsible for this haptic illusion. However, if this haptic illusion is present also in the early blind, explanations founded on visual experience and visualization are invalidated. Henceforth, nonvisual explanations must be found to account for the existence of the same illusion in both vision and touch. These explanations may be common to both sense modalities, but they may also be specific to each of them, since different causes can have the same effects. Finally, one should determine whether the tactual processes implemented by blindfolded sighted subjects are different from those implemented by the early and late blind. If the same results are observed in the three groups, the existence of general haptic processes independent of the visual status of the subjects will be favored. On the other hand, if the early blind have consistently different results, as compared with the two other populations, it will suggest that they implement original haptic processes.

The analysis of the literature about haptic illusions reveals that most of the scenarios above have been observed, depending on the type of illusion studied. The aim of the present study is to show, through an analysis of the contemporary literature, that certain special features of touch allow at least a partial understanding of why contradictory results have been observed and why touch is sometimes as sensitive as vision to certain illusions and sometimes less sensitive to others. These special features may be the exploratory movements at work when a raised figure is perceived by touch. However, in order to do that, let us first recall the general characteristics of touch, as compared with vision.

\section{Touch and Its Characteristics}

Touch differs from vision in that it is a contact modality whose receptors are spread all over the body. As a result, the tactual perceptual field is consistently smaller than the visual perceptual field. Whereas this visual field - that is, the portion of space that activates neural processes at a given moment- has a stable shape and size (a horizontally elongated and incomplete ellipse), the shape and size of the tactile perceptual field varies according to the mode of exploration adopted by the ob- server. In passive tactile perception, where there is no movement, this field is very small, since it is limited to the surface of the skin in contact with objects. Although some discriminations are, however, possible in this situation, tactual perceptual abilities are limited by lack of movement. This article will not consider passive tactual illusions, because they scarcely have been studied.

Most of the time, voluntary movements must be made. Their amplitude required to contact the whole object varies according to the size of the object to be perceived. In this haptic (tactual-kinesthetic) perception, the size of the perceptual field varies according to the body segments activated (a finger, a whole hand, both hands associated with arm movements, etc.). When only a finger is in action, external reference cues are drastically reduced, whereas egocentered cues remain available and may act as an efficient reference frame. Free two-handed exploration enlarges the haptic perceptual field and facilitates the perception of contextual cues (for discussions on the role of reference cues, see Millar, 1994; Millar \& Al-Attar, 2000, 2002). However, enlarging the haptic perceptual field by the activation of all the fingers does not mean that all the sensory data provided are processed simultaneously. Loomis, Klatzky, and Lederman (1991) compared the performances of sighted and blindfolded adults in the identification of raised-line drawings. In the haptic condition, the participants could explore either with one index finger only or with two index fingers. In the visual condition, the participants looked at the drawing through a tube whose diameter equated the size of the visual field with the size of the haptic field when one index finger or two index fingers were used. Results showed that when the visual field corresponded to the haptic field of one index finger, there was no difference between visual and haptic performances. But when the visual field corresponded to the haptic field of two index fingers, visual performances improved, whereas haptic performances did not. This means that the capacity of parallel processing of touch in this complex task is reduced to the information provided by one index finger only.

Kinesthetic information resulting from exploratory movements provides external reference cues too and is associated to purely cutaneous information. These two types of information form a whole known as haptic perception (Gibson, 1962, 1966; Revesz, 1950). Because manual exploration relies on intentional movements specialized according to the property of the objects to be perceived (Lederman \& Klatzky, 1987, 1993), spatial haptic perception may be incomplete and less structured than visual perception. Although exploratory movements are needed in vision too, haptic exploration and haptic processing are consistently more sequential than are visual ones. Consequently, working memory is heavily loaded, and a mental synthesis is necessary to gain a unified representation of the object (Revesz, 1950).

The central role played by movement means that the most mobile areas and the areas most endowed with sensory receptors are the most efficient in the tactual domain. 
Therefore, the hands - or more specifically, the arm-hand systems-are concerned the most.

Because manual haptic perception provides information concerning the spatial properties of the environment, it is largely redundant with respect to vision. Under certain conditions, it allows access to the spatial properties of texture, shape, size, location (direction and distance), orientation, and so forth. However, in most circumstances, it is less efficient than vision in the spatial domain, because the final tactual percept depends on the quality of the exploratory movements carried out, the way in which input is processed, and the mental synthesis made at the end of exploration.

How do these modality-specific characteristics of the haptic system allow one to understand the presence or absence of visual illusions in the haptic modality? Three special features of the way an object is explored and, consequently, the way haptic input is coded plays a role in the presence or absence of geometrical haptic illusions. (1) During exploration, the size of the perceptual field varies depending on whether the subject uses the inside face of an index finger only, the whole hand, or both hands. In the first case, the lines that induce the visual error might not be perceived by the hand, and the haptic illusion may not appear. (2) The intervention of the kinesthetic information resulting from large exploratory movements (when the arm-hand system is involved in the perception of a large stimulus) could produce spatial distortions (Faineteau, Gentaz, \& Viviani, 2003; Klatzky, 1999; Lederman, Klatzky, \& Barber, 1985; Lederman, Klatzky, Collins, \& Wardell, 1987). (3) The gravitational cues generated by the antigravity forces allowing the arm to be kept in the air may provide reference cues not present in the visual illusion (Gentaz \& Hatwell, 1996, 1998, 1999; Heller, Calcaterra, Burson, \& Green, 1997).

We will now examine three visual illusions in detail and will look at the literature to find out in what conditions the presence or absence of these illusions has been evidenced in the haptic domain. Our aim is to find out whether the three above-mentioned factors can explain some of the contradictory results observed. More specifically, we will provide data showing that when they are at work, the haptic modality-specific processes rely more or less directly on the characteristics of haptic exploration.

\section{The Study of Three Geometrical Haptic Illusions}

The analysis will be focused mainly on the following three illusions: the Müller-Lyer illusion, the verticalhorizontal illusion, and the Delboeuf illusion. The reason for this choice is twofold. First, most of the literature on haptic illusions has been concentrated on these figures, and therefore, a convergent set of studies is available in this field. Second and more important, we will see that each of these three illusions illustrates one aspect of the theories outlined above. Some other illusions (Poggendorff, Ponzo, and Oppel-Kundt) will be discussed more briefly in the conclusion of this article, in order to evaluate the general value of our interpretation.
The Müller-Lyer illusion. In the Müller-Lyer illusion, the evaluation of the length of a line segment is modified according to the orientation of the arrowheads situated at both ends. Thus, the length of the line segment with outward-pointing arrowheads is overestimated, as compared with the one (of identical length) with inwardpointing arrowheads. The procedure allowing the measurement of the presence and the intensity of this illusion is generally the following. The two segments are presented side by side (Figure 1A). One of them is the standard, and its length is fixed, whereas the length of the other can vary. The acute angle formed by each arrowhead and the horizontal segment varies according to study but is most often $45^{\circ}$ or $30^{\circ}$.

In vision, the two figures are drawn in black on white paper, whereas in the haptic modality, they are in relief. The linear relief is obtained by thermoform, or lines of raised dots, or small three-dimensional bars. Heller, Brackett, Wilson, Yoneyama, et al. (2002) compared the size estimates of raised lines and of smooth wooden dowels in blindfolded sighted adults and did not observe any difference between the two types of display. In the comparison method, the participant must indicate, after having explored the two figures visually or haptically (blindfolded), whether the variable segment is longer or shorter than the standard segment. In the adjustment method, the participant adjusts the variable segment so that it appears to be the same length as the standard segment.

The results showed that the illusion is present both visually and haptically in blindfolded sighted subjects and in early- and late-blind subjects (Casla, Blanco, \& Travieso, 1999; Hatwell, 1960; Heller, Brackett, Wilson, Yoneyama, et al., 2002; Millar \& Al-Attar, 2002; Over, 1966a, 1966b; Patterson \& Deffenbacher, 1972; Rudel \& Teuber, 1963;

(A)

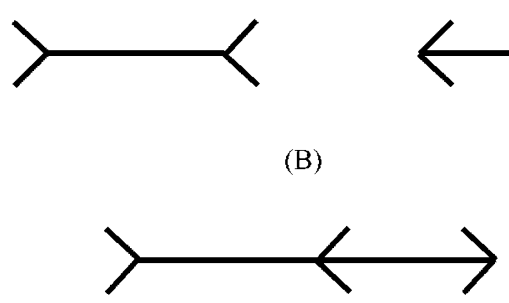

(C)
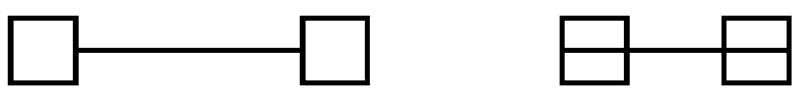

Figure 1. Müller-Lyer illusion. (A) The classical stimuli. (B) The stimuli proposed by Rudel and Teuber (1963). (C) The stimuli proposed by Hatwell (1960). The evaluation of the length of a segment is modified according to the orientation of arrowheads situated at both ends. Thus, the length of the line segment with outward-pointing arrowheads is overestimated, as compared with the one (of identical length) with inward-pointing arrowheads. 
Suzuki \& Arashida, 1992; Tsai, 1967; Wong, 1975). In most of these studies, the subject was told to explore the segment and the arrowheads with the index finger of the dominant hand only. In this case, the intensity of the illusion is the same in the two modalities, the segment with the outward-pointing arrowheads being judged to be 1.2 1.3 times longer than the one with the inward-pointing arrowheads (Suzuki \& Arashida, 1992).

Heller, Brackett, Wilson, Yoneyama, et al. (2002) compared the intensity of this illusion in blindfolded sighted, late-blind, congenitally blind, and low-vision adults, using a new display. The subjects were asked to use their right index fingers to feel the raised-line figure and, simultaneously, were to use their left hands to make size estimates with a tangible sliding ruler. The data showed the presence of the same robust haptic Müller-Lyer illusion in the four populations. The lack of effect of visual status means that the Müller-Lyer illusion in haptics is not dependent on visual experience or visual imagery. These findings are at variance with those of Tsai (1967), who also observed the Müller-Lyer illusion in haptics but found, in addition, more errors in the early-blind subjects than in the lateblind ones. However, because there were very few subjects in each group ( 3 congenitally blind, 3 blinded during childhood, and 3 blinded as adults), this result should be considered cautiously. No difference according to visual status was found by Casla et al. (1999) in blind adults and by Hatwell (1960) in blind children. However, when the subject was asked to freely explore the stimulus with two hands, Hatwell (1960) obtained an illusion weaker in the haptic modality than in the visual modality in a mixed group of early and late totally blind children.

Other researchers have shown that the three factors that act upon the intensity of the visual illusion have the same effect on the haptic illusion. First, Rudel and Teuber (1963) found that, although the subjects received no feedback, the magnitude of errors in the visual and haptic illusions decreased similarly as the number of trials increased (Figure 1B). Over (1967) confirmed this decrease in haptics. This shows that the perceptual learning occurring very generally in vision is also at work in haptics. Second, the error gets stronger as the acute angles formed by the arrowheads and the segment to be evaluated get smaller in both vision (Coren \& Girgus, 1978) and haptics (Heller, Brackett, Wilson, Yoneyama, et al. 2002). Over (1966a) also observed a similar effect of angle on the illusion intensity in both modalities. Hatwell (1960) reported an analogous effect by showing that if, instead of arrowheads, the segments to be evaluated were ended by small squares (in one case, these squares appear to lengthen the segment by forming a $90^{\circ}$ angle with it; in the other, they intersected the segment end part; Figure 1C), the visual error was maintained, whereas the haptic error disappeared. She explained this result by the fact that the $90^{\circ}$ angle formed by the segment and the vertical side of the end square is big enough for the finger to manage to partially exclude the square from the tactile perceptual field. As a result, the effect of the error-inducing lines is de- creased or completely canceled. This exclusion is obviously impossible to achieve in the visual modality.

Third, Millar and Al-Attar (2002) showed that the same experimental manipulations reduce the Müller-Lyer illusion in vision and haptics to the same level of percentage of error. More precisely, detailed explicit instructions to ignore the arrowheads because they are confusing and to use body-centered cues for spatial reference reduce the Müller-Lyer illusion in both modalities to near zero. This illusion is not reduced in the absence of instructions to use body-centered cues, even when external reference cues are present. It should be noted, however, that Millar and Al-Attar (2002) presented the two figures on the tabletop one above the other, instead of side by side, as had been done in previous studies. Whether this particular presentation has an effect on the reference cues used and the magnitude of the haptic illusion is not known.

Finally, Gentaz, Camos, Hatwell, and Jacquet (2004) observed, in provisional results, a positive correlation between the magnitudes of the illusion in the visual and the haptic modalities when the same blindfolded sighted subjects performed exactly the same task in both modalities.

Taken together, these findings constitute strong evidence that the Müller-Lyer illusion involves similar factors in both modalities. The fact that this illusion is present in the haptic modality calls into question purely visual explanations, such as Gregory's "inappropriate adjustment of constancy" (1998). According to this author, the visual error appears when distance is not perceived correctly, due to the presence, in the two-dimensional drawing, of misleading depth cues, such as perspective or textured background. In order to validate this theory, Gregory and Harris (1975) showed, by using an ingenious experimental system, that the visual Müller-Lyer illusion disappears when the visual cues for perspective and depth of the stimulus are adequate. However, the presence of the illusion in the early-blind people who have no visual representation invalidates the explanations founded solely on visual experience and visualization. It rather suggests the presence of analogous haptic processes, independent of the visual status of the subjects. Furthermore, since three factors (learning, angle, and reference instructions) affect the intensity of the visual and the haptic illusions in the same way, the presence of the Müller-Lyer illusion in vision and haptics seems to result from similar visual and haptic processes.

The vertical-horizontal illusion. In the visual verticalhorizontal illusion, the length of the vertical segment is overestimated, as compared with the same segment in a horizontal orientation. Actually, this illusion can be decomposed into two illusions: the vertical illusion observed in L shapes (the pure vertical-horizontal illusion; Figure 2B) and the bisection illusion observed when one segment is bisected whereas the other is not, as in the inverted-T figure (Figure 2A).

The vertical-horizontal illusion has been found in the haptic modality in blindfolded sighted subjects, as well as in the early and late blind (Casla et al., 1999; Day \& Wong, 
1971; Deregowski \& Ellis, 1972; Hatwell, 1960; Heller, Brackett, Wilson, Yoneyama, \& Boyer, 2002; Heller et al., 1997; Heller \& Joyner, 1993; Millar, 2001; Suzuki \& Arashida, 1992; Von Collani, 1979; Wong, 1977). For example, Suzuki and Arashida found that in the visual and haptic modalities, the vertical segment of an inverted-T figure is perceived as being 1.2 times longer than the horizontal segment.

Research has shown that the factors responsible for the presence of the bisection illusion and the variations of its intensity are similar in the visual and the haptic modalities. In both, the overestimation of the vertical segment is higher in the inverted-T figure than in the L figure, where the two segments are undivided (Figure 2B). In the latter case, the illusion is not present either in young blind subjects (Hatwell, 1960) freely exploring the figure or in blindfolded sighted adults (Millar \& Al-Attar, 2000, Experiment 2) exploring with the right index finger of one hand an L figure in which the vertical line is aligned with the body midline. In addition, it is the horizontal line that is overestimated when the inverted-T figure is rotated by $90^{\circ}$ (Figure 2C), which divides the vertical segment in the middle, whereas the horizontal segment is undivided (Hatwell, 1960; Millar \& Al-Attar, 2000, Experiment 3). According to Millar and Al-Attar (2002, Experiment 5), this reduction of illusion in the L figure and its suppression when there is no junction point can be explained by the fact that this junction point acts as an an-

(A)

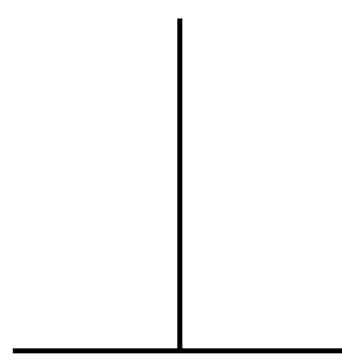

(C)

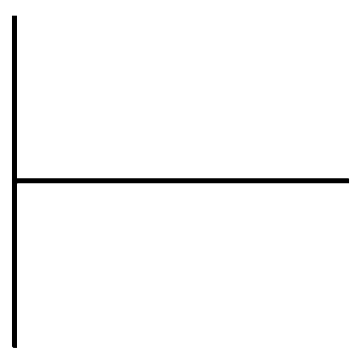

Figure 2. Vertical-horizontal illusion. (A) An inverted-T stimulus. (B) An L stimulus. In both figures, the length of the vertical segment is overestimated, as compared with the same segment in a horizontal orientation. (C) A $90^{\circ}$-rotated inverted-T stimulus. In this figure, the length of the horizontal segment is overestimated, as compared with the same segment in a vertical orientation. chor that is added to the anchors constituted by the ends of the segments.

The importance of the role of the method of exploration has been emphasized by Hatwell (1960), who observed an increase in error with the inverted-T figure when, instead of a free two-handed exploration, blind children were constrained to use only the index finger of the dominant hand. She suggested that these results may be explained by the metric function of the hand (Revesz, 1950), because the fingers are able to act as measurement units when they are allowed to move freely. On the other hand, Millar and AlAttar (2000, Experiments 2 and 6) compared the magnitude of the haptic inverted-T illusion in blindfolded sighted subjects according to whether exploration was restricted to the index finger of the right hand or was two-handed with reference cues available. In the two-handed condition, the subjects were asked to use both hands to relate the feeling of a rigid frame surrounding the stimulus figure to the feeling of the vertical line of the figure, using similar downward movements. In addition, the subjects were instructed to relate the downward scanning movements to their body midline and to the vertical sides of the frame. By contrast, in the one index finger condition, there was no external frame of reference during scanning, and the subjects were asked to explore the whole figure with their index finger. A clear reduction in error in two-handed exploration was observed. The authors attributed this reduction to the instruction to refer to the spatial reference frame, which was available in the two-handed exploration, whereas it was absent when only the index finger of one hand was used. Taken together, these results highlight both the role of bisection, which acts in the same way in vision as in haptics, and the role of reference frames.

By contrast, the factors responsible for the presence of the vertical illusion are specific to each modality. In vision, numerous studies have shown that the error is due to an anisotropy of the visual field (see Prinzmetal \& Gettleman, 1993). According to this theory (first proposed by Künnapas, 1955, 1957a, 1957b, 1957c), this illusion derives from a kind of framing effect. The closer a line extends toward a surrounding frame, the longer it appears to be. Because of the shape of the retina, the visual field is a horizontally oriented ellipse (it subtends approximately $200^{\circ} \times 130^{\circ}$ along the horizontal and the vertical meridians, respectively); vertical lines will generally be closer to the boundary of the visual field than will be the horizontal lines, and hence, vertical lines will appear to be longer. Considerable evidence is consistent with this purely visual explanation of the illusion (for a review, see Prinzmetal \& Gettleman, 1993). On the other hand, in the haptic modality, studies have shown the role of exploratory movements. More precisely, Day and Avery (1970), Deregowski and Ellis (1972), and Wong (1977) have highlighted the role of the direction of movement. In most studies that have revealed the presence of this illusion in haptics, the figure was placed flat on a table. In this position, the exploratory movement is radial for the "vertical" segment (which is, in fact, in the sagittal plane) and tangential for the horizon- 
tal segment. A movement is radial when it develops in a radius having the subject at its center and tangential when it adopts one of the tangents to these radii. Yet Cheng (1968) and Wong (1977) have shown that there is a tendency to overestimate the length of a radial movement, as compared with a tangential movement, because radial movements are executed more slowly than tangential ones. Estimates concerning the lengths of movements are, therefore, affected by time cues. In order to evaluate the role played by the direction of the exploratory movements in the vertical-horizontal haptic illusion, Day and Avery (1970) and Deregowski and Ellis (1972) presented the inverted-T and the L figures in the subject's frontoparallel plane. In this case, all the exploratory movements were tangential. The vertical segments were no longer overestimated in the L figure, since the two factors (radiall tangentialmovement and bisection) could not act. However, the overestimation of the vertical segment (although attenuated) remained present in an inverted-T figure, since it is due to the sole factor of bisection.

Regarding the comparison between the rate of haptic illusion observed in blindfolded sighted, early-blind, and late-blind subjects, the only reported difference between these groups was that in Heller and Joyner's (1993) study. In this research, the size of the stimuli was estimated by the pincer posture of the index finger and the thumb of the subject. In this condition, the early-blind subjects showed similar overestimation in the $\mathrm{L}$ and the inverted-T figures, whereas the late-blind manifested a much greater overestimation of the inverted-T figure. The authors concluded that visual imagery or visual experience was implied in this illusion. However, a further study by Heller, Brackett, Wilson, Yoneyama, and Boyer (2002, Experiment 1) revealed that the above-mentioned results were due to the mode of response used-that is, the thumb-index finger pincer posture. In the latter research, the subjects felt the raised-line patterns with the index finger of one hand and used a tangible sliding ruler with the other hand for size estimates. In this condition, no effect of visual status was observed. The authors concluded that the difference between early- and late-blind people, reported in Heller and Joyner's study, was linked to the response measure and, possibly, to differences in motor skills. It is likely that early-blind people are less trained than the sighted in the use of pincer postures since, in their grasping actions, sighted people are guided by vision during hand prepositioning before the hand grasps an object.

Finally, Heller et al. (1997) suggested that the verticalhorizontal haptic illusion is the result of a nonoptimum haptic exploration of the stimulus. They studied the effects of the type of movement and the implication of whole-arm movements in the presence of the illusion in the inverted-T figure. For example, small shapes can be explored with index finger tracing movements, whereas large stimuli necessitate more ample movements, including whole-arm radial movements, which can lead to perceptual errors. Much of the early research in this field did not take into account the impact of extending the arm or the implication of the whole arm in the presence of the error. Furthermore, the stimulus has often been presented in such a way that the subjects had to keep their elbows on the table during exploration or, in other studies, they had to keep the elbows suspended in the air.

In a first experiment, Heller et al. (1997) examined the effect of maintaining the elbow above the surface of the table. In this case, the illusion was reduced or eliminated, whereas it was present and of great amplitude when the subjects kept their elbows in the air. In a second experiment, arm movements were controlled. One group had to proceed uniquely by fingertip tracing, since the arm and the elbow were immobilized in a drainpipe. In contrast, another group had to use whole-arm movements, since the arm and the hand were placed in a system that isolated the index finger and the arm and that prevented fingertip tracing of the stimulus. The results supported the idea that the presence of the illusion is more likely when whole-arm movements are possible. This shows the importance of scale in haptic perception. When the stimuli are quite small, they can be explored with the fingers. When they are bigger, they necessitate whole-arm movements in order to be explored. Haptic illusions are more likely to occur in this case, according to Heller and his colleagues. Whole-arm movements probably increase the impact of the gravitational cues present during exploration, and Gentaz and Hatwell (1996, 1998) have shown the importance of these cues in haptic perception of spatial orientation in blindfolded sighted subjects and in the early and late blind.

What is the theoretical implication of these results? A common factor, bisection, is observed here, which affects the visual and the haptic modalities in the same way. But contrary to the Müller-Lyer illusion, there are also factors specific to each system: The exploratory movements affect only the haptic illusion, just as the anisotropy of the visual field has an effect only on vision. The presence of the vertical illusion in the haptic modality, including its presence in the early blind, invalidates the exclusively visual explanations of this error. Actually, this illusion is due to one perceptive factor common to the two modalities (bisection), whereas modality-specific factors, resulting in analogous errors, are also responsible for the illusion in each system.

The Delboeuf illusion. In the Delboeuf illusion (Figure 3 ), the evaluation of the size of a circle is modified if it is inserted into an exterior concentric circle. In the visual modality, Piaget (1957) has shown that the inner circle (B) is overestimated as compared with an isolated reference circle $(\mathrm{C})$, when the $\mathrm{B}: \mathrm{A}$ ratio is close to $3: 4$ (A is the exterior concentric circle). More precisely, the illusion is maximum for $\mathrm{B}$ values such as $\mathrm{A}-\mathrm{B}=\mathrm{B} / 6$, and the illusion is negative when $\mathrm{A}-\mathrm{B}=1.7 \mathrm{~B}$ approximately. Hatwell (1960) has shown that, in the haptic modality and in free two-handed exploration, this illusion is not observed in blind children (mixed group of early and late blind) even in conditions under which it is maximum in vision. More recently, Suzuki and Arashida (1992) have confirmed that in the same blindfolded sighted adults, the illusion 


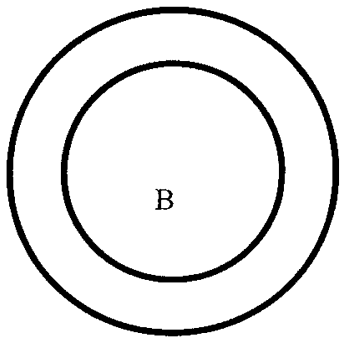

A

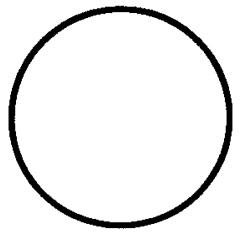

$\mathrm{C}$
Figure 3. Delboeuf illusion. The inner circle $(B)$ is perceived as being larger than the identical isolated circle $(C)$, because it is inserted in an exterior concentric circle (A).

concerning the inner circle (B) is present in vision but absent in the haptic modality. However, curiously, an illusion concerning the exterior circle $(\mathrm{A}$; underestimation of the exterior circle) appears in both vision and touch, with the same amplitude. It must be noted that the latter authors are the only ones to have measured the haptic illusion of the exterior circle of the Delboeuf figure.

On a theoretical level, the fact that this visual illusion is not observed in haptics does not invalidate purely visual interpretations. The absence of this illusion in the haptic modality (if confirmed) may be explained by some features of the exploratory hand movements that permit context effects to be reduced. Thus, in a free exploration situation (Hatwell, 1960) or when only exploration with the index finger of one hand is allowed (Suzuki \& Arashida, 1992), the subjects can use only the internal face of the index finger. Circle B is perceptively isolated when exploration starts at the center of the concentric circles, and Circle A disappears from the haptic perceptive field. The task is thus reduced to a simple comparison of $\mathrm{B}$ and $\mathrm{C}$, in which A plays no role. This interpretation is plausible but it does not explain why the illusion occurs haptically for the exterior circle. In order to test the role played by the exploratory procedure, exploratory movements should be recorded, and the distance between A and B should be varied in order to modify the role of context during exploration. However, too small a gap would be too close to the limit of the haptic discrimination of two points, and Circles A and B would not be differentiated. Whatever it may be, this (partial) absence of the illusion in the haptic modality confirms the analytical nature of haptic apprehension, which is less susceptible to deformations because it can totally isolate one element in order to compare it with another.

\section{General Discussion}

We have seen that the Müller-Lyer illusion is present in vision and in the haptic modality and seems to be the result of similar processes in the two modalities. The vertical-horizontal illusion also exists in vision and haptics but is due partly to similar processes (the role of bisection) and partly to processes specific to each modality (anisotropy due to the shape of the visual field and overestimation of radial exploratory movements, as compared with tangential movements). Finally, the Delboeuf illusion seems to occur only in vision because, haptically, the contextual part of the figure that induces the error may be excluded from the perceptual tactile field of the exploring index finger. This exclusion is impossible in vision, where the interaction between the two concentric circles results in an overestimation of the inner one, probably through a process of assimilation. Therefore, the presence of this illusion in vision and its absence in haptics may be explained by the specific processes at work in each modality.

Furthermore, it is interesting to note that no consistent difference between blindfolded sighted subjects and earlyor late-blind ones has been observed in almost all the studies in which these three populations have been compared. Therefore, neither visual experience nor intense haptic training modifies the haptic processing at work when figures inducing visual illusions are perceived.

The existence of certain visual illusions in the haptic modality for blindfolded sighted and early-blind subjects invalidates or restricts the field of application of purely optical explanations of the visual illusions, as well as those based on visualization. The results observed are, thus, rather in favor of explanations based on the general characteristics of the functioning of the perceptual systems, independent of the sensory modalities. However, these general explanations do not necessarily account for the same illusions. Clearly, it is not enough to study vision in order to understand the other senses, and a systematic study of each modality is necessary.

The role of manual exploratory movements in the presence, the intensity, or the absence of an illusion in the haptic modality has been apparent in many of the studies cited in our analysis, particularly in Hatwell (1960), Heller et al. (1997), and Wong (1997), whereas in Millar and Al-Attar (2000), variations of exploratory conditions have been associated with instructions concerning spatial reference frames. Variations in the size of the haptic perceptual field - according to whether the subject uses an index finger only, both hands in free exploration, or the whole stretched arm-in fact isolate the troublemaking context, bring in an exterior spatial reference frame, or introduce time and gravity cues into the estimation of radial and tangential lengths. Therefore, haptic modality-specific processes are closely related to exploratory movements, and these movements help us to understand why some visual illusions are present in haptics and others are not.

The obvious question is whether our interpretation remains valid for other haptic illusions not examined here. These other illusions have not been studied often in haptics, and the results of these works, which concern only blindfolded sighted subjects, have sometimes been contradictory. For example, the visual Oppel-Kundt illusion (Figure 4A) has been tested in haptics only by Suzuki and Arashida (1992). In this figure, the filled space is visually overestimated, as compared with the unfilled one, although the two spaces are identical. The same error was observed by Suzuki and Arashida in a haptic condition in which the blind- 
(A)

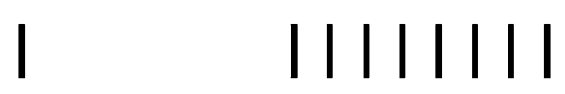

(B)

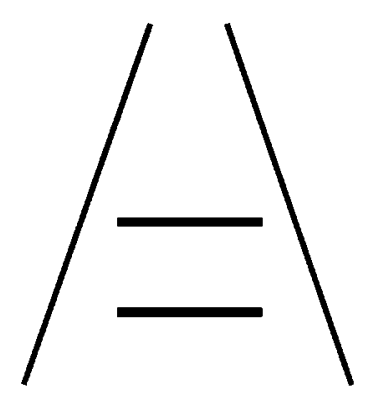

(C)

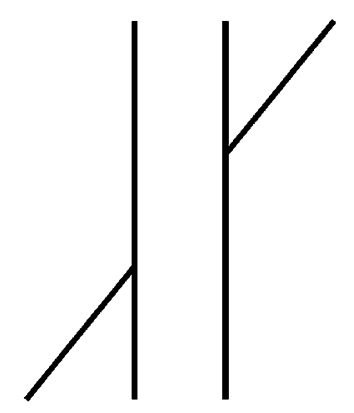

Figure 4. (A) In the Oppel-Kundt illusion, the filled space is perceived as being longer than the unfilled space, although both spaces have the same length. (B) In the Ponzo illusion, the horizontal line nearer the point of convergence is perceived as being longer than the horizontal line that is farther from this point. (C) In the Poggendorff illusion, the oblique line, which seems to be masked by the two vertical lines, is perceived as broken: Its right part seems to be higher than its left part.

folded subjects were not allowed to use thumb-index finger evaluations of the lengths. This thumb-index finger evaluation would have prevented the tactile perception of the vertical bars of the filled space. Therefore, since the lines inducing the visual error were necessarily perceived tactually by the subjects tested by Suzuki and Arashida, the haptic illusion was observed, as in vision. The Ponzo illusion (Figure 4B) has been studied in haptics both by Casla et al. (1999) and by Suzuki and Arashida. Visually, the upper horizontal bar, which is nearer the converging lines, is perceived as being longer than the (identical) lower bar, which is farther from these converging lines. This visual error is probably due to misleading perspective and depth cues. In haptics, the results of the two available studies are contradictory: Whereas Suzuki and Arashida observed the same illusion in haptics, this illusion was absent in Casla et al.'s study. Further research is therefore needed to clarify this point. However, following the logic of our interpretation, this length distortion should not be present or only weakly present in the haptic modality, because it concerns a length estimation in which the finger, if it is free to act, can very easily isolate the segment to be evaluated without perceiving its troublemaking context.

The visual Poggendorff illusion (Figure 4C) has been more often studied in haptics. In vision, the oblique line seems to be masked by the two parallel vertical lines, and it appears as broken. In haptics, Fisher (1966) obtained the same significant error, but his method was questionable because the figure was first presented visually to blindfolded subjects. With a completely haptic presentation, this illusion was not found either by Suzuki and Arashida (1992) or by Wenderoth and Alais (1990), despite a variation in exploratory movements (two or three fingers, elbow and wrist immobilized or free). By contrast, a reversed illusion emerged in Lucca, Dellantonio, and Riggio's study (1986). Whatever it may be, in this visual illusion, the error does not concern length estimation but, rather, the perception of the continuity of an oblique line intersected by two vertical parallel straight lines. Since the Gestalt laws of organization of figures are not always found with the same intensity in the haptic modality as in vision (Hatwell, Orliaguet, \& Brouty, 1990), the interaction between the parallel lines and the oblique one may not be present in touch. Furthermore, thorough research should allow these phenomena to be verified.

In summary, this review of the literature concerning haptic illusions has shown that some of the illusions present in vision are observed also in the haptic modality, but not all of them. Our argument was that these differences stemmed, in great part, from the particularfeatures of hand exploratory movements and, especially, from the fact that the size of the haptic perceptual field may be varied voluntarily by the subject. These variations may result in the perceptual exclusion of the misleading lines. The role of reference frame, evidenced mainly by Millar and Al-Attar (2000, 2002), has been studied only in the Müller-Lyer and the verticalhorizontal illusions, and further research is needed to evaluate how it acts on the other illusions. Research has shown that even when the same illusion is observed in vision and haptics, it does not necessarily result from the same causes. Finally, how these similar processes are implemented in the brain is not yet completely known, and further studies should clarify this issue.

It is noteworthy that there are both theoretical and practical reasons to study haptic perceptual illusions (Millar, 2001). Indeed, the blind depend largely on touch to access spatial and graphic information. We have seen that haptic perception is sensitive to certain perceptual illusions in a way that can be different from that for visual perception. Consequently, relief drawings, raised maps, and all sorts of graphic illustrations intended for the blind should take this factor into account. 


\section{REFERENCES}

BEAN, C. (1938). The blind have "optical" illusions. Journal of Experimental Psychology, 22, 283-289.

Casla, M., Blanco, F., \& Travieso, D. (1999). Haptic perception of geometric illusions by persons who are totally congenitally blind. Journal of Visual Impairment \& Blindness, 93, 583-588.

Cheng, M. (1968). Tactile-kinesthetic perception of length. American Journal of Psychology, 81, 74-82.

Coren, S., \& GIRGus, J. (1978). Seeing is deceiving: The psychology of visual illusions. Hillsdale, NJ: Erlbaum.

Cornoldi, C., Bertuccelli, B., Rocchi, P., \& Sbrana, B. (1993). Processing capacity limitations in pictorial and spatial representations in the totally congenitally blind. Cortex, 29, 675-689.

DAY, R., \& AVERY, G. (1970). Absence of the horizontal-vertical illusion in haptic space. Journal of Experimental Psychology, 83, 172-173.

DAY, R., \& WONG, T. (1971). Radial and tangential movement directions as determinants of the haptic illusion in an L figure. Journal of Experimental Psychology, 87, 19-22.

DEREGOWSKI, J., \& ELLIS, H. D. (1972). Effect of stimulus orientation upon haptic perception of the horizontal-vertical illusion. Journal of Experimental Psychology, 95, 14-19.

Eagleman, D. M. (2001). Visual illusion and neurobiology. Nature Neuroscience Review, 2, 920-926.

FAineteau,H., GentaZ, E., \& ViViani, P. (2003). The kinaesthetic perception of Euclidean distance: A study of the detour effect. Experimental Brain Research, 152, 166-172.

FISHE R, G. H. (1966). A tactual Poggendorff illusion. Nature, 212, 105 106.

Gentaz, E. Camos, V., Hatwell, Y., \& JACQUeT, A.-Y. (2004). The visual and haptic Müller-Lyer illusions. Manuscript submitted for publication.

Gentaz, E., \& Hatwell, Y. (1996). Role of gravitational cues in the haptic perception of orientation. Perception \& Psychophysics, $\mathbf{5 8}$, 1278-1292.

Gentaz, E., \& Hatwell, Y. (1998). The haptic oblique effect in the perception of rod orientation by blind adults. Perception \& Psychophysics, 60, 157-167.

Gentaz, E., \& HATwell, Y. (1999). Role of memorisation conditions in the haptic processing of orientations and the "oblique effect." British Journal of Psychology, 90, 377-388.

GentaZ, E., \& Hatwe Ll, Y. (2002). Le toucher, un sens trompeur? Pour La Science, 293, 78-83.

Gibson, J. J. (1962). Observations on active touch. Psychological Review, 69, 477-490.

GiBson, J. J. (1966). The senses considered as perceptual systems. Boston: Houghton Mifflin.

GrEGORY, R. L. (1998). Eye and brain: The psychology of seeing (5th ed.). London: Oxford University Press.

GrEGORY, R. L., \& HARRIS, J. (1975). Illusion-destruction by appropriate scaling. Perception, 4, 203-220.

HATwELL, Y. (1960). Étude de quelques illusions géométriques tactiles chez les aveugles. L'Année Psychologique, 1, 11-27.

HATWELL, Y. (1978). Form perception and related issues in blind humans. In R. Held, H. Leibowitz, \& H. Teuber (Eds.), Handbook of sensory physiology: VII. Perception (pp. 488-519). New York: SpringerVerlag.

Hatwell, Y. (1985). Piagetian reasoning and the blind (Pierre Verdet, Trans.). New York: American Foundation for the Blind. (Original work published 1966)

Hatwell, Y. (2003). Psychologie cognitive de la cécité précoce. Paris: Dunod.

Hatwell, Y., Orliaguet, J.-P., \& Brouty, G. (1990). Effects of object properties, attentional constraints and manual exploratory procedures on haptic perceptual organization: A developmental study. In H. Bloch \& B. I. Bertenthal (Eds.), Sensory-motor organizations and development in infancy and early childhood (pp. 315-335). Dordrecht: Kluwer.

Hatwell, Y., Streri, A., \& Gentaz, E. (Eds.) (2000). Toucher pour connaître: Psychologie cognitive de la perception tactile manuelle. Paris: Presses Universitaires de France.
Hatwell, Y., Streri, A., \& Gentaz, E. (Eds.) (2003). Touching for knowing. Amsterdam: John Benjamins.

Heller, M. A. (ED.) (2000). Touch, representation and blindness. Oxford: Oxford University Press.

Heller, M. A., Brackett, D. D., Wilson, K., Yoneyama, K., \& BOYER, A. (2002). Visual experience and the haptic horizontalvertical illusion. British Journal of Visual Impairment, 20, 105-109.

Heller, M. A., Brackett, D. D., Wilson, K., Yoneyama, K., BoYer, A., \& STEFFEN, H. (2002). The haptic Müller-Lyer illusion in sighted and blind people. Perception, 31, 1263-1274.

Heller, M. A., Calcaterra, J. A., Burson, L. L., \& Green, S. L. (1997). The tactual horizontal-vertical illusion depends on radial motion of the entire arm. Perception \& Psychophysics, 59, 1297-1311.

Heller, M. A., \& JoYner, T. D. (1993). Mechanisms in the haptic horizontal-vertical illusion: Evidence from sighted and blind subjects. Perception \& Psychophysics, 53, 422-428.

KLATZKY, R. L. (1999). Path completion after haptic exploration without vision: Implications for haptic spatial representations. Perception \& Psychophysics, 61, 220-235.

KüNNAPAS, T. M. (1955). An analysis of the "vertical-horizontal illusion.” Journal of Experimental Psychology, 49, 134-140.

KÜNNAPAS, T. M. (1957a). Interocular differences in the verticalhorizontal illusion. Acta Psychologica, 13, 253-259.

KÜNNAPAS, T. M. (1957b). Vertical-horizontal illusions and surrounding field. Acta Psychologica, 13, 35-42.

KüNNAPAS, T. M. (1957c). The vertical-horizontal illusion and the visual field. Journal of Experimental Psychology, 53, 405-407.

LEDERMAN, S. J, \& KLATZKY, R. L. (1987). Hand movements: A window into haptic object recognition. Cognitive Psychology, 19, 342-368.

LEDERMAN, S. J., \& KLATZKY, R. L. (1993). Extracting object properties through haptic exploration. Acta Psychologica, 84, 29-40.

Lederman, S. J., KlatzKy, R. L., \& BARber, P. O. (1985). Spatial and movement-based heuristics for encoding pattern information through touch. Journal of Experimental Psychology: General, 114, 33-49.

Lederman, S. J., Klatzky, R. L., Collins, A., \& WARdell, J. (1987). Exploring environments by hand or foot: Time-based heuristics for encoding distance in movement space. Journal of Experimental Psychology: Memory \& Language, 16, 606-614.

Loomis, J. M., KlatzKy, R. L., \& Lederman, S. J. (1991). Similarity of tactual and visual picture recognition with limited field of view. Perception, 20, 167-177.

Lucca, A., Dellantonio,A., \& Riggio, L. (1986). Some observations on the Poggendorff and Müller-Lyer tactual illusions. Perception \& Psychophysics, 39, 374-380.

MILLAR, S. (1994). Understanding and representing space: Theory and evidence from studies with blind and sighted children. Oxford: Oxford University Press, Clarendon Press.

Millar, S. (2001). Illusions in reading maps by touch: Reducing distance errors. British Journal of Psychology, 92, 643-657.

Millar, S., \& Al-ATtAR, Z. (2000). Vertical and bisection bias in active touch. Perception, 29, 481-500.

Millar, S., \& Al-ATTAR, Z. (2002). The Müller-Lyer illusion in touch and vision: Implications for multisensory processes. Perception \& Psychophysics, 64, 353-365.

OVER, R. (1966a). A comparison of haptic and visual judgments of some illusions. American Journal of Psychology, 79, 590-595.

OVER, R. (1966b). Context and movement as factors influencing haptic illusions. Australian Journal of Psychology, 3, 262-265.

OvER, R. (1967). Intermanual transfer of practice decrements with a haptic illusion. Quarterly Journal of Experimental Psychology, 19, 215-218.

Patterson, J., \& DefFenbacher, K. (1972). Haptic perception of the Müller-Lyer illusion by the blind. Perceptual \& Motor Skills, 35, 819824.

Piaget, J. (1957). Les mécanismes perceptifs. Paris: Presses Universitaires de France.

PICK, H. (1974). Visual coding of non-visual spatial information. In R. MacLeod \& H. Pick (Eds.), Perception (pp. 153-165). Ithaca, NY: Cornell University Press.

PrinzMETAL, W., \& GetTleman, L. (1993). Vertical-horizontal illusion: One eye is better than two. Perception \& Psychophysics, 53, 81-88. 
Revesz, G. (1934). System der optischen und haptischen Raumtäuschungen. Zeitschrift für Physiologie, 131, 296-375.

RE VESZ, G. (1950). Psychology and art of the blind. London: Longmans Green.

Rudel, R., \& Teuber, H.-L. (1963). Decrement of visual and haptic Müller-Lyer illusion on repeated trials: A study of cross-modal transfer. Quarterly Journal of Experimental Psychology, 15, 125-131.

SUZUKI, K., \& ARASHIDA, R. (1992). Geometrical haptic illusions revisited: Haptic illusions compared with visual illusions. Perception \& Psychophysics, 52, 329-335.

Thinus-Blanc, C., \& GAUnet, F. (1997). Representation of space in the blind: Vision as a spatial sense? Psychological Bulletin, 121, 20-42.

TSAI, L. S. (1967). Müller-Lyer illusion by the blind. Perceptual \& Motor Skills, 25, 641-644.

VON COLlani, G. (1979). An analysis of illusion components with $L$ and perpendicular figures in active touch. Quarterly Journal of Experimental Psychology, 31, 241-248.
Wenderoth, P., \& Alais, D. (1990). Lack of evidence for a tactual Poggendorff illusion. Perception \& Psychophysics, 48, 234-242.

WONG, T. S. (1975). The respective role of limb and eye movements in the haptic and visual Müller-Lyer illusion. Quarterly Journal of Experimental Psychology, 27, 659-666.

WONG, T. S. (1977). Dynamic properties of radial and tangential movements as determinants of the haptic horizontal-vertical illusion with an L figure. Journal of Experimental Psychology: Human Perception \& Performance, 3, 151-164.

WorCHE L, P. (1951). Space perception and orientation in the blind. Psychological Monographs: General \& Applied, 65, (15, Whole No. 332), 1-28.

(Manuscript received July 12, 2002; revision accepted for publication April 1, 2003.) 